\title{
Initial management of traumatic brain injury in the rural setting
}

\author{
Stephen Honeybul, Paul Woods ${ }^{1}$ \\ Departments of Neurosurgery, ${ }^{1}$ Intensive Care Medicine, Sir Charles Gairdner Hospital and Royal Perth Hospital, Western Australia
}

\section{ABSTRACT}

Healthcare workers in the rural setting face unique problems when dealing with head injured patients however the basic principle of medical management are the same in any situation. The key initial elements remain aggressive early resuscitation followed by a comprehensive assessment of conscious level and either early consultation or transfer to a neurosurgical facility. What has improved considerably over recent years is the understanding of the pathophysiology of traumatic brain injury and as such some of the medical management strategies have changed. A basic understanding of some of these concepts is useful in the clinical setting and serves to emphasis the importance of effective early medical management. Thereafter consideration must be given to which patients require radiological investigations and possible discussion with or transfer to a neurosurgical facility.

Key words: Initial management, traumatic brain injury, rural trauma

\section{Introduction}

Western Australia is unique in its geographical isolation. It is the state is the largest in Australia covering 2.5 million kilometers' in total with a population of approximately 2.2 million. The vast majority of patients with traumatic brain injury that are transferred from remote and regional areas are taken to one of the two neurotrauma centres in the Perth metropolitan area. Between them these centres admit approximately four hundred neurotrauma cases per year and whilst most cases originate in the metropolitan region there are approximately twenty cases per year that are transferred from remote and rural regions These transfers are often performed by the Royal Flying Doctor Service of Australia (RFDS) and whilst the average flight covers approximately $500 \mathrm{kms}$ it can be as far as 3,000 kms with overall transfer times being up to twelve hours. As such healthcare workers in the rural

\begin{tabular}{|l|l|}
\hline \multicolumn{2}{|c|}{ Access this article online } \\
\hline Quick Response Code: & Website: \\
\hline & www.ruralneuropractice.com \\
\cline { 2 - 3 } & \\
\hline & DOI: \\
\hline
\end{tabular}

setting face unique problems when dealing with these injured patients however the basic principles of medical management are the same in any situation. The key elements remain aggressive early resuscitation followed by a comprehensive assessment of conscious level and either early consultation or transfer to a neurosurgical facility. What has improved considerably over recent years is the understanding of the pathophysiology of traumatic brain injury and as such some of the basic management strategies have changed. An understanding of these concepts is useful in the rural clinical setting and the aim of this review is to present the current guidelines for the rural medical management of traumatic brain injury in Western Australia.

\section{The pathophysiology of traumatic brain injury}

Traditionally primary brain injury has been defined as occurring at the moment of impact and secondary injury follows thereafter and consists of a wide range of ischaemic, ionic, neurochemical and inflammatory insults. However it is now realised that there is considerable overlap between the two processes and a substantial amount of cell death that occurs many hours later is due to a series of deleterious neurochemical cascades processes that are initiated at the time of injury. ${ }^{[1,2]}$ This concept is well illustrated by the glutamate neuroexcitatory cascade ${ }^{[3]}$ which is one such response

Address for correspondence:

Dr. Honeybul S, Department of Neurosurgery, Sir Charles Gairdner Hospital and Royal Perth Hospital, Western Australia.

E-mail: stephen.honeybul@health.wa.gov.au 
that is initiated at the time of injury and amplified by the well-known secondary insults of hypoxia and hypotension [Figure 1]. ${ }^{[4]}$

When the brain is injured and starts to swell or there is a mass lesion such as an intracerebral haematoma the compensation is made at the expense of blood and CSF volume. As the brain becomes progressively more swollen or a mass lesion increases in size these compensatory mechanisms become exhausted and for incrementally smaller increases in volume there are progressively greater increases in pressure [Figure 2].

Understanding these basic concepts is important in order to appreciate why the management of traumatic brain injury has evolved over recent years.

\section{Initial management of head injury}

The immediate management of the severely head injured patient is surprisingly simple and is focused on preventing secondary insults by a timely and thorough initial resuscitation in order to maintain cerebral perfusion and avoid ischaemia. ${ }^{[4]}$ Thereafter an accurate and reproducible assessment of neurological function is required.

\section{Initial resuscitation}

There are clear and concise guidelines regarding the initial management and these are well described in the ATLS and the APLS protocols. The importance of maintaining a clear, secure airway and assuring adequate ventilation cannot be over stated. The cervical spine injury must be protected and initial fluid resuscitation should be with colloid or crystalloid solutions. Hypotonic and glucose containing fluids must be avoided ${ }^{[5]}$ Hypertonic saline is now used routinely in neurointensive care ${ }^{[6]}$ however

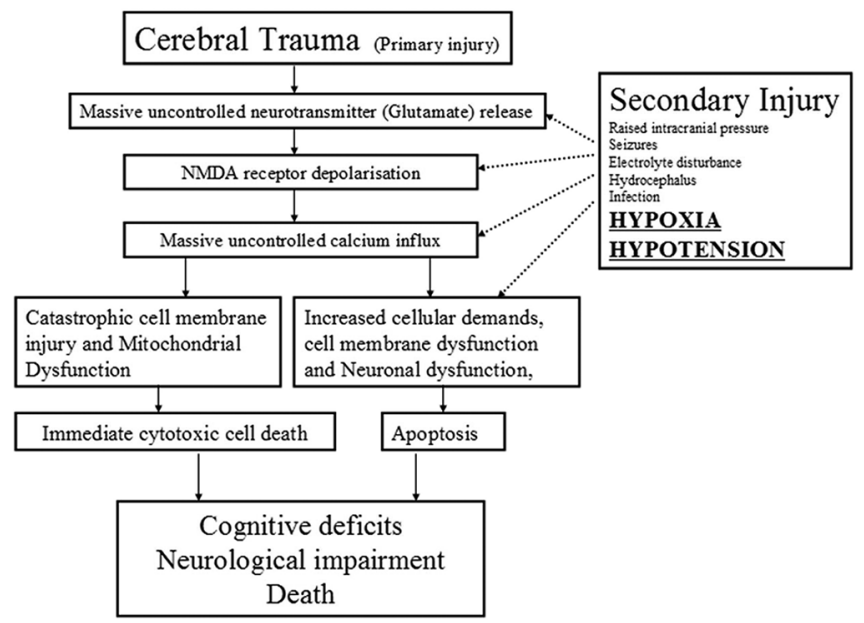

Figure 1: The neuroexcitatory cascade. The primary injury triggers a massive uncontrolled release of the neurotransmitter glutamate which triggers the cascade leading to cytotoxic and apoptotic cell death. The cascade is reinforced by secondary insults in the rural setting this may not be available. If this is the case then isotonic fluids are recommended.

\section{Neurological assessment}

Having initiated measures for the primary resuscitation and identified and managed any immediately life threatening injuries a neurological assessment is required. This is combined with an examination of the pupils and a blood glucose level.

- Pupil reaction: In the unconscious patient a dilated pupil is a sign of the cerebral swelling and represents incipient brainstem compression which may require the use of mannitol and urgent neurosurgical consultation. In the conscious, alert patient unequal pupils are more likely to be either a premorbid finding or due to a direct injury to the eye.

- Blood glucose level: Whilst this forms part of the neurological assessment it can be performed at any stage of the initial resuscitation (for example, when venous access is obtained), placing this test at specific point is useful if only so that it is not forgotten. Hypoglycaemia must obviously be recognised early and treated promptly.

- Glasgow coma score [Figure 3]: The Glasgow Coma Score (GCS) is used to provide an accurate and reliable baseline neurological assessment of conscious level..$^{[7]}$ This is important because:

i. It documents the baseline level of neurological injury

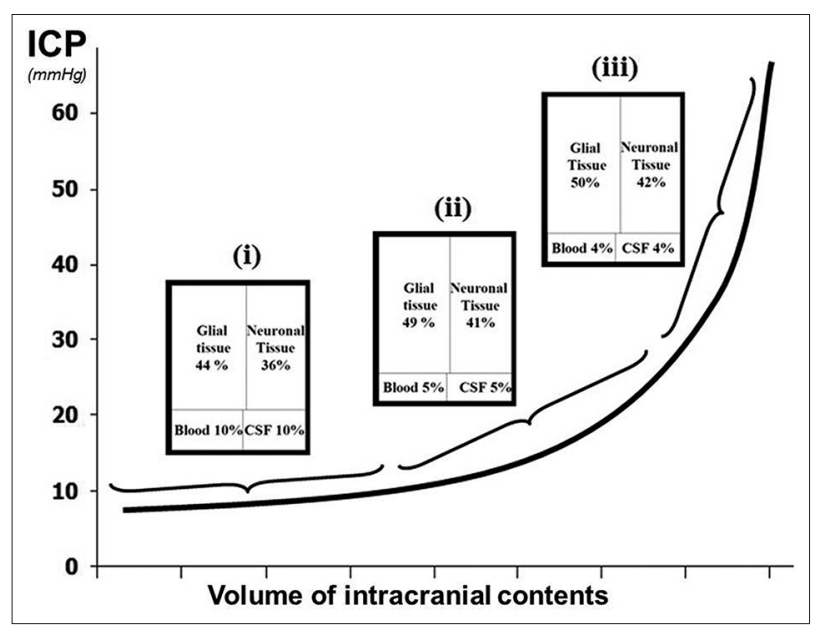

Figure 2: (i) Normal physiology - Any increase in volume of the constituent components of the intracranial compartment does not cause a significant increase in ICP because of compensatory decrease in volume of either blood or CSF; (ii) As the brain progressively swells initial compensation is at the expense of blood and CSF; (iii) As the cerebral swelling worsens or a mass lesion enlarges there comes a point when the compensatory mechanisms start to fail and for smaller increases in swelling there are incrementally greater increases in intracranial pressure 
ii. It can identify any change in conscious level.

This especially important in the context of a patient with an extradural haematoma in which there is an initial "lucid" period followed some hours later by a precipitous decline in conscious level as the haematoma expands.

iii. It is important when considering prognosis.

The baseline GCS provides a useful measure of the severity of the primary brain injury which is important when considering long term outcome

What must always be remembered is that whilst a "score" can be generated it was developed as a scale and the findings should always be presented as the individual scores. There are three neurological responses to be considered:

i. The motor response: Amongst the three categories the motor response provides the most important prognostic significance. ${ }^{[8]}$ Whilst the difference between obeying commands and localising is represented by only a single point it represents the most important recording within the whole GCS and signifies a huge deterioration in conscious level. Figure 4 illustrates the importance of providing a breakdown of the assessment. The assessment of case 2 gives a much clearer picture of the actual neurological function which can be clearly and concisely communicated.

ii. Verbal response: Whilst this category is named the verbal response it must always be remembered that the GCS is a measure of conscious level and not of focal neurological function. If a patient cannot verbalise due to a focal deficit, a tracheostomy or a maxillofacial injury, they can always communicate whether they are orientated or not by giving

\section{Conscious level Assessment}

Assessment

\section{Glasgow Coma Scale}

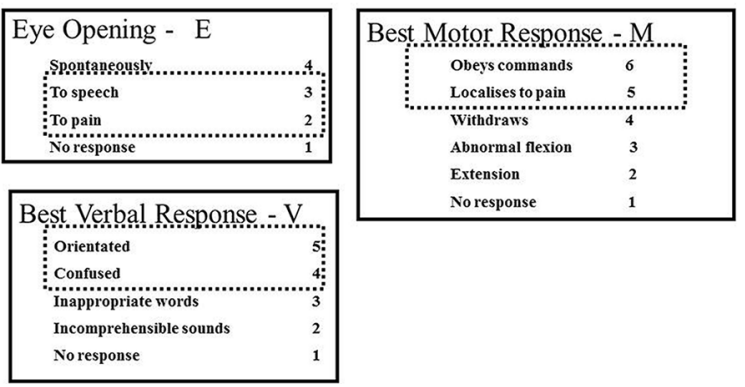

Figure 3: The glasgow coma scale. The dotted boxes highlight the most important aspects of the assessment non-verbal messages. For example, a patient could ask to nod if asked to name the correct year and can then be given a number of options. This reemphasises the importance of using the breakdown of the GCS as a measure on consciousness rather than a simple number. If a patient is able to verbalise the most important distinction is whether they are orientated or not and this is easy to determine by using simple non ambiguous questions such as: Where are you, what day is it, what year is it. If these are answered correctly the patient is broadly orientated to time and place. A more detailed assessment in the field is not required.

iii. Eye opening response: Whilst this category has the least prognostic significance it is a useful measure of subtle changes in conscious level and for this reason the manner in which the assessment is performed is important. The correct manner is to approach the patient and if they are not eye opening spontaneously ask them in a normal voice volume to open their eyes. The vocal volume can then be increased and if there is no response a mild tactile stimulation such as rubbing the shoulder may be used. This can then be followed by a painful stimulus such as a sternal rub.

By using this approach, the GCS can provide a detailed assessment of conscious level that can be assessed in a consistent reproducible manner and can be reliably communicated between healthcare professionals with differing levels of expertise. This is fundamentally important because only by accurately assessing conscious level, changes can be identified and acted upon. The assessments must be commenced in the field and continued regularly thereafter, so the trends will are identified.

\begin{tabular}{|l|}
\hline Case 1. \\
52 Female motorcyclist MVA \\
GCS $12-14$ \\
PERL \\
Major pelvic and lower limb \\
fractures \\
\begin{tabular}{|l} 
Case $2 .$. \\
42 male assault, GCS $12-14$ \\
• Consistently squeezes fingers with his right hand (6 points), \\
he remains hemiplegic on the left hand side (allways \\
record the higher level of function) \\
• Occasionally confused to date and year ( 4 - 5 points) \\
- Most of the time he will eye open to voice howere he \\
Occasionally requires a mild painful stimuli (2-3 points) \\
PERL, Lower limb fractures
\end{tabular} \\
\hline
\end{tabular}

Figure 4: Two clinical cases used to illustrate the importance of providing a breakdown of the GCS. In case 2, a much clearer and reproducible assessment is provided 


\section{Hyperventilation, barbiturates and hypothermia}

For many years these therapies formed the cornerstone of the management of patients with traumatic brain injury. Patients were routinely hyperventilated, ${ }^{[9,10]}$ placed in a barbiturate coma ${ }^{[11,12]}$ and more recently rendered hypothermic. ${ }^{[12]}$ However examining the basic principles of the Monroe Kellie doctrine demonstrates why these measures are used less frequently. There is no doubt that barbiturates and hypothermia have the potential to be neuroprotective due to their influence on many mechanisms known to be important in the cellular response to injury. ${ }^{[11,12]}$ However the mechanism by which the intracranial pressure is lowered is by cerebral vasoconstriction [Figure 5]. Reducing the cellular metabolic rate leads to lower oxygen consumption and less carbon dioxide is produced. This induces the physiological response of vasoconstriction which lowers the cerebral blood volume which in turn reduces the intracranial pressure. Unfortunately the last thing the injured brain needs is less blood and many studies that have demonstrated that whilst these measures lower the intracranial pressure they do not improve outcome. ${ }^{[9,13-15]}$ Indeed, both barbiturate therapy and hypothermia have been shown to associated with significant complications most notably, hypotension and pneumonia. ${ }^{[13,15]}$

Although these therapies is no longer used routinely they are still used in life threatening situations as a stop gap method to buy time prior to surgical intervention or for acute rises in intracranial pressure. What must always be considered however is the pathophysiological method by which that time is bought and the possible consequences in terms of cerebral ischaemia.

\section{Ongoing management considerations}

Following initial resuscitation stabilisation and clinical assessment a patient may be transferred to a primary or secondary medical facility. It is here there are a number of issues require consideration:

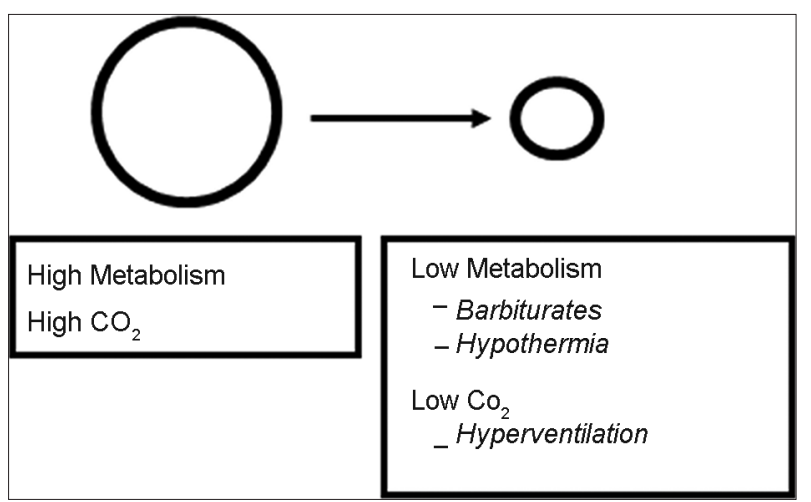

Figure 5: The pathophysiological effects of lowering arterial carbon dioxide. The blood vessel response is vasoconstriction with a subsequent lowering of intracranial pressure

\section{Radiological investigations}

Which patients require a CT scan following head injury is always a source of debate especially in the rural setting where this may involve transfer over considerable distances. ${ }^{[18,19]}$ In many cases the need for investigations is obvious however for mild to moderate head injuries the indications can often be less clear cut and this has been the subject of a number of publications. ${ }^{[18-22]} \mathrm{A}$ few useful guidelines would be:

a. Patients who remain confused two hours after the initial injury

b. Severe headache accompanied by nausea and vomiting

c. Any patient with a focal neurologic deficit

d. A dangerous mechanism such as a high speed motor vehicle accident of fall from a height greater then three feet.

These are merely guideline and there will always be patients who are "just not right" and this may prompt investigation. ${ }^{[22]}$ Plain radiographs have a limited role however in certain circumstance where CT scanning may be unavailable the presence of a linear skull fracture may alert the physician to the possibility and location of an extradural haematoma

\section{Transfer to a neurosurgical centre}

Western Australia is unique in its isolation and in general it is probably better to transfer or seek advice early rather than wait for a clinical change by which time a precipitous deterioration may occur. Given the distances involved a useful rule of thumb would be "if you are thinking about transferring a patient, the patient is probably best transferred". This is obviously not always the case but neurosurgical centres have twenty four hour cover and phone call advice is always

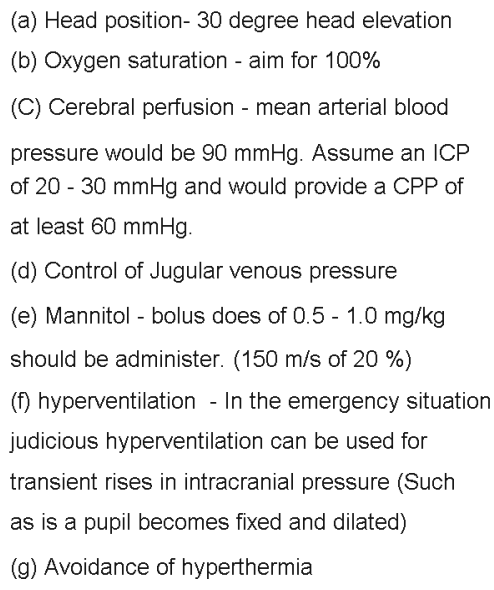

Figure 6: Clinical deterioration. There are a number of measures that can be instituted in a step wise fashion that can maximise cerebral perfusion and avoid secondary brain injury 
available and encouraged. Patients who have the findings listed above or who are in a facility where a CT scan is available and that scan is abnormal, probably need to be either in a neurosurgical facility or in a facility where they can be urgently transferred within the hour.

\section{Seizure management}

Seizures are relatively common in the first twenty four hours following traumatic brain injury and most will resolve in less than a minute. The temptation must be resisted to abort the seizure by giving large doses of benzodiazepines. This usually heavily sedates the patient such that they require intubation. If the seizure continues beyond 60 seconds incremental doses of lorazepam, diazepam or midazolam can be used. Thereafter the patient can be loaded with an anticonvulsant such as Phenytoin or Keppra.

\section{Progressive neurological deterioration}

In the conscious patients deterioration in the GCS or the development of a focal deficit can be a sign of either cerebral swelling or an enlarging intracranial mass lesion In patients with multiple injuries who require intubation and ventilation, whilst the clinical examination may be lost, detailed attention of the pupil reactivity and symmetry provides an early warning of an enlarging mass lesion. In addition, the cardiovascular response of hypertension and bradycardia represent part of the Cushing response and are an ominous sign of rising ICP. In these situations there are a number measures which may be used to temporarily improve matters in the rural setting whilst urgently transferring to a neurosurgical facility. [Figure 6]. ${ }^{[21]}$

\section{Safe evacuation of a head injured patient}

The severely head injured patient should be transported after consultation with the receiving neurosurgical and intensive care teams. The airway should be secure and cerebral oxygenation should be optimised, this may require intubation. Cardiovascular support should be instituted prior to transport if necessary to ensure adequate perfusion pressure.

The most common issues that cause problems that arise in the transport of head injured patients are;

- Delay in arranging transfer

- Inadequate resuscitation before transfer

- Inadequate preparation for the journey

- Inadequate care during the journey.

Movement of the severely head injured patient should be planned, smooth, expedient and supervised by adequately experienced personnel.

\section{Conclusions}

Head injury remains a very significant contributor to mortality and morbidity from blunt trauma especially in young people. Whilst treatment strategies continue to evolve the basic medical management is based on adequate resuscitation, accurate and reproducible assessment and prevention of secondary brain injury.

\section{References}

1. Sahuquillo J, Poca MA, Amoros S. Current aspects of pathophysiology and cell dysfunction after severe head injury. Curr Pharm Des 2001;7:1475-503.

2. Reilly PL. Brain injury: The pathophysiology of the first hours. 'Talk and Die revisited'. J Clin Neurosci 2001;8:398-403.

3. Nicholls DG, Ward MW. Mitochondrial membrane potential and neuronal glutamate excitotoxicity: Mortality and millivolts. Trends Neurosci 2000;23:166-74.

4. Manley G, Knudson MM, Morabito D, Damron S, Erickson V, Pitts L. Hypotension, hypoxia, and head injury: Frequency, duration, and consequences. Arch Surg 2001;136:1118-23.

5. Ertmer C, Kampmeier T, Rehberg S, Lange M. Fluid resuscitation in multiple trauma patients. Curr Opin Anaesthesiol 2011;24:202-8.

6. Simma B, Burger R, Falk M, Sacher P, Fanconi S. A prospective, randomized, and controlled study of fluid management in children with severe head injury: Lactated Ringer's solution versus hypertonic saline. Crit Care Med 1998;26:1265-70.

7. Teasdale G, Jennett B. Assessment and prognosis of coma after head injury Acta Neurochir (Wien) 1976;34:45-55.

8. Steyerberg EW, Mushkudiani N, Perel P, Butcher I, Lu J, McHugh GS, et al. Predicting outcome after traumatic brain injury: Development and international validation of prognostic scores based on admission characteristics. PLoS Med 2008;5:e165.

9. Curley G, Kavanagh BP, Laffey JG. Hypocapnia and the injured brain: More harm than benefit. Crit Care Med 2010;38:1348-59.

10. Heffner JE, Sahn SA. Controlled hyperventilation in patients with intracranial hypertension. Application and management. Arch Intern Med 1983;143:765-9.

11. Rea GL, Rockswold GL. Barbiturate therapy in uncontrolled intracranial hypertension. Neurosurgery 1983;12:401-4.

12. Sahuquillo J, Vilalta A. Cooling the injured brain: How does moderate hypothermia influence the pathophysiology of traumatic brain injury. Curr Pharm Des 2003;13:2310-22.

13. Eisenberg HM, Frankowski RF, Contant CF, Marshall LF, Walker MD. High-dose barbiturate control of elevated intracranial pressure in patients with severe head injury. J Neurosurg 1988;69:15-23.

14. Hutchison JS, Ward RE, Lacroix J, Hébert, PC, Barnes MA, Bohn DJ, et al. Hypothermia Pediatric Head Injury Trial Investigators and the Canadian Critical Care Trials Group. Hypothermia therapy after traumatic brain injury in children. N Engl J Med 2008;5:2447-56.

15. Clifton GL, Valadka A, Zygun D, Coffey CS, Drever P, Fourwinds S, et al. Very early hypothermia induction in patients with severe brain injury (the National Acute Brain Injury Study: Hypothermia II): A randomised trial. Lancet Neurol 2011;10:131-9.

16. Abdul Latip LS, Ahmad Alias NA, Ariff AR, Shuaib IL, Abdullah J, Naing NN. CT scan in minor head injury: A guide for rural doctors. Clin Neurosci 2004;11:835-9.

17. Simpson DA, Worth RJ. Neurotrauma in country hospitals: The role of computerized tomography scanning. Aust N Z J Surg 1989;59:1-3.

18. Ingebrigtsen T, Romner B, Kock-Jensen C. Scandinavian guidelines for initial management of minimal, mild, and moderate head injuries. The Scandinavian Neurotrauma Committee. J Trauma 2000;48:760-6.

19. Bratton SL, Chestnut RM, Ghajar J, McConnell Hammond FF, Harris OA, Hartl R, et al. Brain Trauma Foundation; American Association of Neurological Surgeons; Congress of Neurological Surgeons; Joint Section 
on Neurotrauma and Critical Care, AANS/CNS. Guidelines for the management of severe traumatic brain injury. J Neurotrauma 2007;24:S83-6.

20. Stiell IG, Wells GA, Vandemheen K, Clement C, Lesiuk H, Laupacis A, et al. The Canadian CT Head Rule for patients with minor head injury. Lancet 2001;357:1391-6

21. Abdul Latip LS, Ahmad Alias NA, Ariff AR, Shuaib IL, Abdullah J, Naing NN. CT scan in minor head injury: A guide for rural doctors. J Clin Neurosci 2004;11:835-9.
22. Easter JS, Grossman SA, Woodruff MM, Rosen CL. When the rules do not work: Head injury without loss of consciousness. J Emerg Med 2008;35:77-80.

How to cite this article: Honeybul S, Woods P. Initial management of traumatic brain injury in the rural setting. $J$ Neurosci Rural Pract 2013;4:33-8.

Source of Support: Nil. Conflict of Interest: None declared. 